\title{
Unifying two results of Orlov on singularity categories
}

\author{
Xiao-Wu Chen
}

Received: 22 March 2010 / Published online: 12 October 2010

(C) Mathematisches Seminar der Universität Hamburg and Springer 2010

\begin{abstract}
Let $\mathbb{X}$ be a separated Noetherian scheme of finite Krull dimension which has enough locally free sheaves of finite rank and let $U \subseteq \mathbb{X}$ be an open subscheme. We prove that the singularity category of $U$ is triangle equivalent to the Verdier quotient triangulated category of the singularity category of $\mathbb{X}$ with respect to the thick triangulated subcategory generated by sheaves supported in the complement of $U$. The result unifies two results of Orlov. We also prove a noncommutative version of this result.
\end{abstract}

Keywords Singularity category · Quotient functor · Schur functor

Mathematics Subject Classification $18 \mathrm{G} 35 \cdot 14 \mathrm{~B} 05 \cdot 16 \mathrm{E} 65$

\section{Introduction}

Let $\mathbb{X}$ be a separated Noetherian scheme of finite Krull dimension which has enough locally free sheaves of finite rank. The last assumption means that any coherent sheaf on $\mathbb{X}$ is a quotient of a locally free sheaf of finite rank ([8, Chap. III, Ex. 6.4]). Denote by coh $\mathbb{X}$ the category of coherent sheaves on $\mathbb{X}$ and by $\mathbf{D}^{b}(\operatorname{coh} \mathbb{X})$ the bounded derived category of coh $\mathbb{X}$. We will identify coh $\mathbb{X}$ as the full subcategory of $\mathbf{D}^{b}(\operatorname{coh} \mathbb{X})$ consisting of stalk complexes concentrated at degree zero.

Communicated by B. Siebert.

This project was supported by Alexander von Humboldt Stiftung and National Natural Science Foundation of China (No. 10971206).

X.-W. Chen ( $₫)$

Department of Mathematics, University of Science and Technology of China, Hefei 230026, China

e-mail: xwchen@mail.ustc.edu.cn

url: http://mail.ustc.edu.cn/ xwchen

Present address:

X.-W. Chen

Institut für Mathematik, Universität Paderborn, 33095 Paderborn, Germany 
Recall that a complex in $\mathbf{D}^{b}(\operatorname{coh} \mathbb{X})$ is perfect provided that locally it is isomorphic to a bounded complex of locally free sheaves of finite rank ([1]). Under our assumption, perfect complexes are equal to those complexes which are isomorphic to a bounded complex of locally free sheaves of finite rank; see [12, Remark 1.7]. Denote by perf $\mathbb{X}$ the full triangulated subcategory of $\mathbf{D}^{b}$ (coh $\mathbb{X}$ ) consisting of perfect complexes; it is a thick subcategory, that is, it is closed under taking direct summands (by [13, Proposition 1.11]).

For the scheme $\mathbb{X}, \mathrm{D}$. Orlov introduced in [12] a new invariant, called the singularity category of $\mathbb{X}$, which is defined to be the Verdier quotient triangulated category $\mathbf{D}_{\mathrm{sg}}(\mathbb{X})=$ $\mathbf{D}^{b}(\operatorname{coh} \mathbb{X}) / \operatorname{perf} \mathbb{X}$. Denote by $q: \mathbf{D}^{b}(\operatorname{coh} \mathbb{X}) \rightarrow \mathbf{D}_{\mathrm{sg}}(\mathbb{X})$ the quotient functor. The singularity category $\mathbf{D}_{\mathrm{sg}}(\mathbb{X})$ captures certain properties of the singularity of $\mathbb{X}$. For example, the scheme $\mathbb{X}$ is regular if and only if its singularity category $\mathbf{D}_{\mathrm{sg}}(\mathbb{X})$ is trivial. Singularity categories are closely related to the Homological Mirror Symmetry Conjecture; see [12-15].

We will explain two results of Orlov which we are going to unify. Let $j: U \hookrightarrow \mathbb{X}$ be an open immersion. Note that the inverse image functor $j^{*}: \operatorname{coh} \mathbb{X} \rightarrow \operatorname{coh} U$ is exact and then it extends to a triangle functor $\mathbf{D}^{b}(\operatorname{coh} \mathbb{X}) \rightarrow \mathbf{D}^{b}(\operatorname{coh} U)$, which is still denoted by $j^{*}$. This functor sends perfect complexes to perfect complexes and then it induces a triangle functor $\bar{j}^{*}: \mathbf{D}_{\mathrm{sg}}(\mathbb{X}) \rightarrow \mathbf{D}_{\mathrm{sg}}(U)$. Denote by $\operatorname{Sing}(\mathbb{X})$ the singular locus of $\mathbb{X}$.

The first result is about a local property of singularity categories; see [12, Proposition 1.14].

Proposition 1.1 (Orlov) Use the notation as above. Assume that $\operatorname{Sing}(\mathbb{X}) \subseteq U$. Then the triangle functor $\bar{j}^{*}: \mathbf{D}_{\mathrm{sg}}(\mathbb{X}) \rightarrow \mathbf{D}_{\mathrm{sg}}(U)$ is an equivalence.

Set $Z=\mathbb{X} \backslash U$ to be the complement of $U$. Denote by $\operatorname{coh}_{Z} \mathbb{X}$ the full subcategory of coh $\mathbb{X}$ consisting of sheaves supported in $Z$. Denote by $\mathbf{D}_{Z}^{b}($ coh $\mathbb{X})$ the full triangulated subcategory of $\mathbf{D}^{b}($ coh $\mathbb{X})$ consisting of complexes with cohomologies supported in $Z$.

For a set $\mathcal{S}$ of objects in a triangulated category $\mathcal{T}$ we denote by thick $\langle\mathcal{S}\rangle$ the smallest thick triangulated subcategory of $\mathcal{T}$ containing $\mathcal{S}$. The subcategory thick $\langle\mathcal{S}\rangle$ of $\mathcal{T}$ is said to be generated by $\mathcal{S}$. For example, we have that $\mathbf{D}_{Z}^{b}(\operatorname{coh} \mathbb{X})=\operatorname{thick}\left\langle\operatorname{coh}_{Z} \mathbb{X}\right\rangle$ (by [7, Chap. I, Lemma 7.2(4)]).

Here is the second result of our interest; compare [15, Proposition 2.7].

Proposition 1.2 (Orlov) Use the notation as above. Assume that $\operatorname{Sing}(\mathbb{X}) \subseteq Z$. Then we have $\mathbf{D}_{\mathrm{sg}}(\mathbb{X})=\operatorname{thick}\left\langle q\left(\operatorname{coh}_{Z} \mathbb{X}\right)\right\rangle$.

The aim of this note is to prove the following result which somehow unifies the two results of Orlov mentioned above. Let us point out that it is in spirit close to a result by Krause ([10, Proposition 6.9]).

Theorem 1.3 Use the notation as above. Then the triangle functor $\bar{j}^{*}: \mathbf{D}_{\mathrm{sg}}(\mathbb{X}) \rightarrow \mathbf{D}_{\mathrm{sg}}(U)$ induces a triangle equivalence

$$
\mathbf{D}_{\mathrm{sg}}(\mathbb{X}) / \operatorname{thick}\left\langle q\left(\operatorname{coh}_{Z} \mathbb{X}\right)\right\rangle \simeq \mathbf{D}_{\mathrm{sg}}(U)
$$

We will see that Theorem 1.3 implies Propositions 1.1 and 1.2 and their converses; see Corollaries 2.3 and 2.4.

We will also prove a noncommutative version of Theorem 1.3, which implies a result in [3] and its converse; see Theorem 3.1 and Corollary 3.3. Note that for a left-Noetherian ring $R$ one has the notion of singularity category $\mathbf{D}_{\mathrm{sg}}(R)$ of $R$, which is defined analogously to 
the singularity category of a scheme. Let us remark that the singularity category $\mathbf{D}_{\mathrm{sg}}(R)$ was studied by Buchweitz in [2] under the name "stable derived category" (compare [6]).

\section{The proof of Theorem 1.3}

In this section we will prove Theorem 1.3.

Recall that for a triangle functor $F: \mathcal{T} \rightarrow \mathcal{T}^{\prime}$ its essential kernel Ker $F$ is a thick triangulated category of $\mathcal{T}$ and then $F$ induces uniquely a triangle functor $\bar{F}: \mathcal{T} / \operatorname{Ker} F \rightarrow \mathcal{T}^{\prime}$. We say that the functor $F$ is a quotient functor provided that the induced functor $\bar{F}$ is an equivalence.

The following lemma is easy.

Lemma 2.1 Let $F: \mathcal{T} \rightarrow \mathcal{T}^{\prime}$ be a quotient functor. Assume that $\mathcal{N} \subseteq \mathcal{T}$ and $\mathcal{N}^{\prime} \subseteq \mathcal{T}^{\prime}$ are thick triangulated subcategories such that $F(\mathcal{N}) \subseteq \mathcal{N}^{\prime}$. Then the induced functor $\mathcal{T} / \mathcal{N} \rightarrow$ $\mathcal{T}^{\prime} / \mathcal{N}^{\prime}$ by $F$ is a quotient functor.

Proof Denote by $\mathcal{N}^{\prime \prime}$ the inverse image of $\mathcal{N}^{\prime}$ under $F$. Since the triangulated subcategory $\mathcal{N}^{\prime}$ of $\mathcal{T}^{\prime}$ is thick, we have that $\mathcal{N}^{\prime \prime}$ is a thick triangulated subcategory of $\mathcal{T}$. Note that $\operatorname{Ker} F \subseteq \mathcal{N}^{\prime \prime}$ and by assumption $\mathcal{N} \subseteq \mathcal{N}^{\prime \prime}$. We identify $\mathcal{T}^{\prime}$ with $\mathcal{T} / \operatorname{Ker} F$ via $\bar{F}$. Then we have equivalences of triangulated categories $\mathcal{T}^{\prime} / \mathcal{N}^{\prime} \simeq \mathcal{T} / \mathcal{N}^{\prime \prime} \simeq(\mathcal{T} / \mathcal{N}) /\left(\mathcal{N}^{\prime \prime} / \mathcal{N}\right)$; see $[16$, Chap. 1, §2, 4-3 Corollaire]. This proves that the induced functor $\mathcal{T} / \mathcal{N} \rightarrow \mathcal{T}^{\prime} / \mathcal{N}^{\prime}$ is a quotient functor.

Let $\mathbb{X}$ be a scheme as in Sect. 1 . Let $j: U \hookrightarrow \mathbb{X}$ be an open immersion and let $Z=\mathbb{X} \backslash U$ be the complement of $U$. The following lemma is well known; compare [15, Lemma 2.2].

Lemma 2.2 The inverse image functor $j^{*}: \mathbf{D}^{b}(\operatorname{coh} \mathbb{X}) \rightarrow \mathbf{D}^{b}(\operatorname{coh} U)$ is a quotient functor with $\operatorname{Ker} j^{*}=\mathbf{D}_{Z}^{b}(\operatorname{coh} \mathbb{X})$.

Proof It is direct to see that $\operatorname{Ker} j^{*}=\mathbf{D}_{Z}^{b}(\operatorname{coh} \mathbb{X})$. Note that the inverse image functor $j^{*}: \operatorname{coh} \mathbb{X} \rightarrow \operatorname{coh} U$ is exact and its essential kernel is $\operatorname{coh}_{Z} \mathbb{X}$. In particular, $\operatorname{coh}_{Z} \mathbb{X} \subseteq \operatorname{coh} \mathbb{X}$ is a Serre subcategory. The functor $j^{*}$ induces an equivalence $\operatorname{coh} \mathbb{X} / \operatorname{coh}_{Z} \mathbb{X} \simeq \operatorname{coh} U$ of abelian categories; compare [4, Chap. VI, Proposition 3]. Here, $\operatorname{coh} \mathbb{X} / \operatorname{coh}_{Z} \mathbb{X}$ is the quotient abelian category in the sense of [4]. Now the result follows immediately from [11, Theorem 3.2].

Proof of Theorem 1.3 By combining Lemmas 2.1 and 2.2 we infer that the functor $\bar{j}^{*}: \mathbf{D}_{\mathrm{sg}}(\mathbb{X}) \rightarrow \mathbf{D}_{\mathrm{sg}}(U)$ is a quotient functor. Then it suffices to show that $\operatorname{Ker} \bar{j}^{*}=$ thick $\left\langle q\left(\operatorname{coh}_{Z} \mathbb{X}\right)\right\rangle$. Since $j^{*}$ vanishes on $\operatorname{coh}_{Z} \mathbb{X}$, we have thick $\left\langle q\left(\operatorname{coh}_{Z} \mathbb{X}\right)\right\rangle \subseteq \operatorname{Ker} \bar{j}^{*}$.

To show that $\operatorname{Ker} \bar{j}^{*} \subseteq$ thick $\left\langle q\left(\operatorname{coh}_{Z} \mathbb{X}\right)\right\rangle$, let $\mathcal{F}^{\bullet}$ be a bounded complex of sheaves lying in $\operatorname{Ker} \bar{j}^{*}$, that is, the complex $j^{*}\left(\mathcal{F}^{\bullet}\right)$ in $\mathbf{D}^{b}(\operatorname{coh} U)$ is perfect. We will show that $\mathcal{F}^{\bullet} \in$ thick $\left\langle q\left(\operatorname{coh}_{Z} \mathbb{X}\right)\right\rangle$. Let us remark that the argument below is almost the same as the one in the proof of [15, Proposition 2.7]. Since $\mathbb{X}$ has enough locally free sheaves of finite rank, there exists a bounded complex $\cdots \rightarrow 0 \rightarrow \mathcal{K}^{-n} \rightarrow \mathcal{E}^{1-n} \rightarrow \mathcal{E}^{2-n} \rightarrow \cdots \rightarrow \mathcal{E}^{m} \rightarrow 0 \rightarrow \cdots$ with each $\mathcal{E}^{j}$ locally free, which is quasi-isomorphic to $\mathcal{F}^{\bullet}$; moreover, we may take $n$ arbitrarily large; see [7, Chap. I, Lemma 4.6(1)]. Here, the coherent sheaf $\mathcal{K}^{-n}$ may not be locally free. By a brutal truncation we have the following distinguished triangle in $\mathbf{D}^{b}(\operatorname{coh} \mathbb{X})$

$$
\mathcal{E}^{\bullet} \longrightarrow \mathcal{F}^{\bullet} \stackrel{\alpha}{\longrightarrow} \mathcal{K}^{-n}[n] \longrightarrow \mathcal{E}^{\bullet}[1]
$$


where $\mathcal{E}^{\bullet}$ denotes the perfect complex $\cdots \rightarrow 0 \rightarrow \mathcal{E}^{1-n} \rightarrow \mathcal{E}^{2-n} \rightarrow \cdots \rightarrow \mathcal{E}^{m} \rightarrow 0 \rightarrow \cdots$. It follows that $q(\alpha)$ is an isomorphism in $\mathbf{D}_{\mathrm{sg}}(\mathbb{X})$. On the other hand, since $j^{*}\left(\mathcal{F}^{\bullet}\right)$ is perfect, taking $n$ large enough we get that $j^{*}(\alpha)$ is zero (in $\mathbf{D}^{b}(\operatorname{coh} U)$ ); see [13, Proposition 1.11]. By Lemma 2.2 this implies that $\alpha$ factors through a complex in $\mathbf{D}_{Z}^{b}(\operatorname{coh} \mathbb{X})$ and then the isomorphism $q(\alpha)$ factors through an object in $q\left(\mathbf{D}_{Z}^{b}(\operatorname{coh} \mathbb{X})\right)$. This implies that $\mathcal{F}^{\bullet}$ is a direct summand of an object in $q\left(\mathbf{D}_{Z}^{b}(\operatorname{coh} \mathbb{X})\right)$. Recall that $\mathbf{D}_{Z}^{b}(\operatorname{coh} \mathbb{X})=\operatorname{thick}\left\langle\operatorname{coh}_{Z} \mathbb{X}\right\rangle$. This implies that $\mathcal{F}^{\bullet} \in \operatorname{thick}\left\langle q\left(\operatorname{coh}_{Z} \mathbb{X}\right)\right\rangle$.

Theorem 1.3 implies Proposition 1.1 and its converse. Recall that $\operatorname{Sing}(\mathbb{X})$ denotes the singular locus of $\mathbb{X}$.

Corollary 2.3 Use the notation as above. Then $\bar{j}^{*}: \mathbf{D}_{\mathrm{sg}}(\mathbb{X}) \rightarrow \mathbf{D}_{\mathrm{sg}}(U)$ is an equivalence if and only if $\operatorname{Sing}(\mathbb{X}) \subseteq U$.

Proof By Theorem 1.3 it suffices to show that $\operatorname{coh}_{Z} \mathbb{X} \subseteq$ perf $\mathbb{X}$ if and only if $\operatorname{Sing}(\mathbb{X}) \subseteq U$. Recall that a coherent sheaf $\mathcal{F}$ on $\mathbb{X}$ is perfect if and only if at each point $x \in \mathbb{X}$, the stalk $\mathcal{F}_{x}$, as an $\mathcal{O}_{\mathbb{X}, x}$-module, has finite projective dimension; see [8, Chap. III, Ex. 6.5]. Then the "if" part follows. To see the "only if" part, assume on the contrary that $\operatorname{Sing}(\mathbb{X}) \nsubseteq U$. Then there is a closed singular point $x \in Z$. The skyscraper sheaf $k(x) \in \operatorname{coh}_{Z} \mathbb{X}$ is not perfect. A contradiction!

Theorem 1.3 implies Proposition 1.2 and its converse.

Corollary 2.4 Use the notation as above. Then $\mathbf{D}_{\mathrm{sg}}(\mathbb{X})=\operatorname{thick}\left\langle q\left(\operatorname{coh}_{Z} \mathbb{X}\right)\right\rangle$ if and only if $\operatorname{Sing}(\mathbb{X}) \subseteq Z$.

Proof Recall that $\mathbf{D}_{\mathrm{sg}}(U)$ is trivial if and only if the scheme $U$ is regular, or equivalently, $\operatorname{Sing}(\mathbb{X}) \subseteq Z$. Then we are done by Theorem 1.3.

We would like to point out that Corollary 2.4 generalizes slightly a result by Keller, Murfet and Van den Bergh ([9, Proposition A.2]). Let $R$ be a commutative Noetherian local ring with its maximal ideal $\mathfrak{m}$ and its residue field $k=R / \mathfrak{m}$. Set $\mathbb{X}=\operatorname{Spec}(R)$ and $U=\mathbb{X} \backslash\{\mathfrak{m}\}$. We identify coh $\mathbb{X}$ with the category of finitely generated $R$-modules, and then $\operatorname{coh}_{\{\mathfrak{m}\}} \mathbb{X}$ is identified with the category of finite length $R$-modules. We infer from Corollary 2.4 that $\operatorname{Sing}(\mathbb{X}) \subseteq\{\mathfrak{m}\}$, that is, the ring $R$ is an isolated singularity if and only if $\mathbf{D}_{\mathrm{sg}}(\mathbb{X})=\operatorname{thick}\langle q(k)\rangle$, that is, the singularity category $\mathbf{D}_{\mathrm{sg}}(\mathbb{X})$ is generated by the residue field $k$.

\section{A noncommutative version of Theorem 1.3}

In this section we will prove a noncommutative version of Theorem 1.3.

Let $R$ be a left-Noetherian ring. Denote by $R$-mod the category of finitely generated left $R$-modules and by $\mathbf{D}^{b}(R$-mod) the bounded derived category of $R$-mod. We denote by $R$-proj the full subcategory of $R$-mod consisting of projective modules and by $\mathbf{K}^{b}(R$-proj) the bounded homotopy category of $R$-proj. We may view $\mathbf{K}^{b}$ ( $R$-proj) as a thick triangulated subcategory of $\mathbf{D}^{b}(R$-mod) (compare [2, 1.2] and [6, 1.4]). Following Orlov ([14]), the singularity category of the ring $R$ is defined to be the Verdier quotient triangulated category 
$\mathbf{D}_{\mathrm{sg}}(R)=\mathbf{D}^{b}(R$-mod $) / \mathbf{K}^{b}(R$-proj $)$; compare [2,6]. We denote by $q: \mathbf{D}^{b}(R$-mod $) \rightarrow \mathbf{D}_{\text {sg }}(R)$ the quotient functor.

Let $e \in R$ be an idempotent, that is, $e^{2}=e$. It is direct to see that $e R e$ is a left-Noetherian ring. Note that $e R$ has a natural $e R e-R$-bimodule structure inherited from the multiplication of $R$. Consider the Schur functor

$$
S_{e}=e R \otimes_{R}-: R-\bmod \longrightarrow e R e-\bmod .
$$

It is an exact functor; see [5, 6.2]. We denote by $\mathcal{B}_{e}$ the essential kernel of $S_{e}$. Note that an $R$-module $M$ lies in $\mathcal{B}_{e}$ if and only if $e M=0$, and if and only if $(1-e) M=M$. The Schur functor $S_{e}$ extends to a triangle functor $\mathbf{D}^{b}(R-\bmod ) \rightarrow \mathbf{D}^{b}(e R e-\bmod )$, which is still denoted by $S_{e}$.

We assume that the left $e R e$-module ${ }_{e R e} e R$ has finite projective dimension. In this case, the functor $S_{e}: \mathbf{D}^{b}\left(R\right.$-mod) $\rightarrow \mathbf{D}^{b}$ (eRe-mod) sends $\mathbf{K}^{b}\left(R\right.$-proj) to $\mathbf{K}^{b}$ (eRe-proj). Then it induces uniquely a triangle functor $\bar{S}_{e}: \mathbf{D}_{\mathrm{sg}}(R) \rightarrow \mathbf{D}_{\mathrm{sg}}(e R e)$. The question on when the functor $\bar{S}_{e}$ is an equivalence was studied in [3].

The following result can be viewed as a (possibly naive) noncommutative version of Theorem 1.3.

Theorem 3.1 Let $R$ be a left-Noetherian ring and let $e \in R$ be an idempotent. Assume that the left eRe-module ${ }_{e R e} e R$ has finite projective dimension. Then the triangle functor $\bar{S}_{e}: \mathbf{D}_{\mathrm{sg}}(R) \rightarrow \mathbf{D}_{\mathrm{sg}}(e R e)$ induces a triangle equivalence

$$
\mathbf{D}_{\mathrm{sg}}(R) / \operatorname{thick}\left\langle q\left(\mathcal{B}_{e}\right)\right\rangle \simeq \mathbf{D}_{\mathrm{sg}}(e R e)
$$

Denote by $\mathcal{N}_{e}$ the full triangulated subcategory of $\mathbf{D}^{b}(R$-mod) consisting of complexes with cohomologies in $\mathcal{B}_{e}$. Note that $\mathcal{N}_{e}=\operatorname{thick}\left\langle\mathcal{B}_{e}\right\rangle$. We will use the following well-known lemma.

Lemma 3.2 ([3, Lemma 2.2]) The triangle functor $S_{e}: \mathbf{D}^{b}\left(R\right.$-mod) $\rightarrow \mathbf{D}^{b}$ (eRe-mod) is a quotient functor with $\operatorname{Ker} S_{e}=\mathcal{N}_{e}$.

Proof of Theorem 3.1 By combining Lemmas 2.1 and 3.2 we infer that the functor $\bar{S}_{e}: \mathbf{D}_{\mathrm{sg}}(R) \rightarrow \mathbf{D}_{\mathrm{sg}}(e R e)$ is a quotient functor. Then it suffices to show that Ker $\bar{S}_{e}=$ thick $\left\langle q\left(\mathcal{B}_{e}\right)\right\rangle$. Then the same proof as the one of Theorem 1.3 will work. We simply replace locally free sheaves of finite rank by projective modules. We omit the details here.

We need some notions. An idempotent $e \in R$ is said to be regular provided that every module in $\mathcal{B}_{1-e}$ has finite projective dimension; $e$ is said to be singularly-complete provided that the idempotent $1-e$ is regular; see [3, Sect. 2].

We have the following immediate consequence of Theorem 3.1, which contains [3, Theorem 2.1] and its converse. Let us point out that this result is quite useful in determining the singularity categories for a certain class of rings; see [3, Sect. 4].

Corollary 3.3 Keep the assumption as above. Then $\bar{S}_{e}: \mathbf{D}_{\mathrm{sg}}(R) \rightarrow \mathbf{D}_{\mathrm{sg}}(e R e)$ is an equivalence if and only if the idempotent $e$ is singularly-complete. 


\section{References}

1. Berthelot, P., Grothendieck, A., Illusie, L.: Théorie des Intersections et Théoreme de Riemmand-Roch. Lecture Notes in Math., vol. 225. Springer, Berlin (1971)

2. Buchweitz, R.O.: Maximal Cohen-Macaulay modules and Tate-cohomology over Gorenstein rings. Unpublished manuscript (1987)

3. Chen, X.W.: Singularity categories, Schur functors and triangular matrix rings. Algebr. Represent. Theory 12, 181-191 (2009)

4. Gabriel, P.: Des catégories abéliennes. Bull. Soc. Math. Fr. 90, 323-448 (1962)

5. Green, J.A.: Polynomial Reprepsentations of $\mathrm{GL}_{n}$, 2nd edn. Lecture Notes in Math., vol. 830. Springer, Berlin (2007)

6. Happel, D.: On Gorenstein algebras. In: Representation Theory of Finite Groups and Finite-dimensional Algebras, Bielefeld, 1991. Prog. Math., vol. 95, pp. 389-404. Birkhäuser, Basel (1991)

7. Hartshorne, R.: Duality and Residue. Lecture Notes in Math., vol. 20. Springer, Berlin (1966)

8. Harshorne, R.: Algebraic Geometry. Graduate Texts in Math., vol. 52. Springer, Berlin (1977)

9. Keller, B., Murfet, D., Van den Bergh, M.: On two examples by Iyama and Yoshino. Compos. Math. (to appear). arXiv:0803.0720v3

10. Krause, H.: The stable derived category of a Noetherian scheme. Compos. Math. 141, 1128-1162 (2005)

11. Miyachi, J.I.: Localization of triangulated categories and derived categories. J. Algebra 141, 463-483 (1991)

12. Orlov, D.: Triangulated categories of singularities and D-branes in Landau-Ginzburg models. Tr. St. Math. Inst. 204, 240-262 (2004)

13. Orlov, D.: Triangulated categories of singularities and equivalences between Landau-Ginzburg models. Mat. Sb. 197, 1827-1840 (2006)

14. Orlov, D.: Derived categories of coherent sheaves and triangulated categories of singularities. In: Algebra, Arithmetic, and Geometry: In Honor of Yu.I. Manin, vol. II. Prog. Math., vol. 270, pp. 503-531. Birkhäuser, Boston (2009)

15. Orlov, D.: Formal completions and idempotent completions of triangulated categories of singularities. Adv. Math. (2010). doi:10.1016/j.aim.2010.06.016. arXiv:0901.1859v1

16. Verdier, J.L.: Categories derivées. In: SGA 4 1/2. Lecture Notes in Math., vol. 569. Springer, Berlin (1977) 